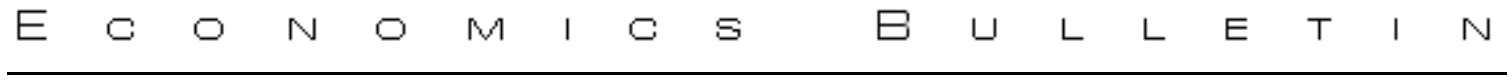

\title{
Stochastic and deterministic menus in common agency games
}

\author{
Gwenael Piaser \\ Universita Ca' Foscari di Venezia
}

\begin{abstract}
In this note, we argue that in Common Agency games the restriction todeterministic menus is critical. We give an simple example, with complete information and no moral hazard, where an equilibrium is not robust to the introduction of stochastic menus.
\end{abstract}

I would like to thank Andrea Attar, Eloisa Campioni, Patrick Leoni, a referee and an associate editor for their comments and suggestions. The usual disclaimers apply.

Citation: Piaser, Gwenael, (2005) "Stochastic and deterministic menus in common agency games." Economics Bulletin, Vol. 4, No. 11 pp. 1-6

Submitted: May 30, 2005. Accepted: September 23, 2005.

URL: http://www.economicsbulletin.com/2005/volume4/EB-05D80017A.pdf 


\section{Introduction}

In games where principals compete in mechanisms, the Revelation Principle does not hold: In general, attention to standard direct mechanisms is not without loss of generality. However, a Delegation Principle is valid. Roughly, the later says that all equilibrium outcomes of any common agency game where many principals can offer arbitrarily complex mechanisms to a single agent, can also be implemented as an equilibrium outcomes of a game where principals are restricted to offer sets (menus) of lotteries over payoff-relevant allocations. (See Peters (2001) and Martimort \& Stole (2002)).

The standard literature on Common Agency games usually assumes that lotteries are not available. This assumption drastically simplifies the analysis, but it carries some unpleasant implications, which have not been fully investigated yet. For example, Martimort and Stole write:

The main restriction in our analysis over general communication mechanism games, therefore, is to limit the principals to offering menus with only deterministic outcomes (i.e., nonlinear price-quantity schedules) rather than allowing for more general menus of distributions (i.e., nonlinear pricequantity lottery schedules). We are not aware of any equilibrium generated by lotteries that is not also generated by nonlinear prices, but at present we cannot state that this restriction is without loss of generality. ${ }^{1}$

The point of this paper is to give simple example with complete information and no moral hazard showing that in a game where players have non-linear preferences some equilibria are not robust to the introduction of lotteries.

Lotteries convexify the strategy set, and the convex hull is typically bigger than the set itself. When facing a strategy profile from other players, any player with non-linear preferences will typically have a different best-response with a larger strategy set. This idea applies to any game with many principals and many agents. ${ }^{2}$

\section{The Model}

We consider a scenario where there are a number of principals (indexed by $i \in N=$ $\{1, \ldots, n\}$ ) contracting with one agent (denoted by index, 0 ). The agent's type is drawn from a set $\Theta$ having a probability distribution $F($.$) that is common knowledge. We$ assume that the agent takes an effort $e$ from the set $E$. The principal $i$ takes an action as

\footnotetext{
${ }^{1}$ Martimort \& Stole (2003a, p. 20, footnote 17)

${ }^{2}$ See Attar et al. (2005) for a discussion on the role of deterministic mechanisms and the Revelation Principle in multiprincipal multiagent models.
} 
well: He has to decide which allocation $y_{i} \in Y_{i}$ should be implemented. A principal is able to contract on a probability distribution over $Y_{i}$. In the following, $\Delta Y_{i}$ denotes the set of all probability distributions over $Y_{i}{ }^{3}$

The payoff to principal $i \in\{1, \ldots N\}$ is represented by the von Neumann-Morgenstern utility function $V_{i}: \prod_{k \in N} Y_{k} \times E \times \Theta \rightarrow \mathbb{R}_{+}$, while the agent's payoff is given by the function $U: \prod_{k \in N} Y_{k} \times E \times \Theta \rightarrow \mathbb{R}_{+}$.

The principals compete through mechanisms. Each principals' mechanism is a couple $\left(M_{i}, \sigma_{i}\right)$ where $M_{i}$ is the chosen message space and $\sigma_{i}$ is a map from $M_{i}$ to $\Delta Y_{i}$. The set of all feasible message spaces will be given by $\mathcal{M}_{i}$ and we also denote $\mathcal{M}=\times_{i \in N} \mathcal{M}_{>}$ and $M=\times_{i \in N} M_{i}$. For the sake of expositional clarity, we assume that $\forall i \in N, \Theta \in \mathcal{M}_{i}$ and $\forall i \in N, \forall T_{i} \subset \Delta Y_{i}, T_{i} \in \mathcal{M}_{i}$. These assumptions do not alter the qualitative insights of the analysis, as long as the sets $\mathcal{M}_{i}$ are rich enough.

Finally, we will use the compact notation $\pi_{i}=\left(M_{i}, \sigma_{i}\right)$. The principal i's strategy set will be given by $\Pi_{i}^{\Delta}$, and $\Pi^{\Delta}$ denotes the collection of all those sets.

Each agent chooses a message $m_{i} \in M_{i}$ for each principal and an effort $e$. For every collection of mechanisms $(\sigma, M) \in \Pi^{\Delta}$, a pure strategy for the agent is given by the map $\sigma_{0}: \Theta \times \Sigma^{\Delta}(M) \rightarrow M \times E$; we will use $\Sigma_{0}$ to denote the agent's strategy set. Given the collection of message spaces $\mathcal{M}$ the common agency game $\Gamma_{\mathcal{M}}^{\Delta}$ is:

$$
\Gamma_{\mathscr{M}}^{\Delta}=\left\{\Theta,\left(\Pi_{i}^{\Delta}\right)_{i \in N}, \Sigma_{0}, U(., ., \theta),\left(V_{i}(., ., \theta)\right)_{i \in N}, F(.)\right\} .
$$

We focus on the pure strategy Perfect Bayesian Equilibrium $(P B E)$ of the game $\Gamma_{\mathscr{M}}^{\Delta}$.

Peters (2001) and Martimort \& Stole (2002) show that even if the Revelation Principle fails to hold with respect to the game $\Gamma_{\mathscr{M}}^{\Delta}$, the so-called Delegation Principle can be applied in a general way. That is:

- One can restrict the set of relevant message spaces and consider the sets of all subsets of $\Delta Y_{i}$ rather than $\mathcal{M}_{i}$.

- It can be assumed that the map $\sigma_{i}$ is the identity over the chosen subset of $\Delta Y_{i}$.

The common agency game where principals are restricted to use menus is given by:

$$
\Gamma_{\Delta Y}=\left\{\Theta,\left(2^{\Delta Y_{i}}\right)_{i \in N}, \bar{\Sigma}_{0}, U(., ., \theta),\left(V_{i}(., ., \theta)\right)_{i \in N}, F(.)\right\}
$$

\footnotetext{
${ }^{3}$ In this model, playing a lottery is a pure strategy for a principal, this point has already been clarified by Peters (2001).
} 
where $2^{\Delta Y_{i}}$ denotes the set of all possible subsets of $\Delta Y_{i}$.

Roughly speaking, the Delegation Principle states that one can consider the game $\Gamma_{\Delta Y}$ rather than the game $\Gamma_{\mathscr{M}}$, which simplifies the analysis.

\section{Deterministic Menus}

If lotteries are not allowed, then a menu for principal $i$ is given subset $T_{i}$ of the decision space $Y_{i}$. We argue that there is no direct relationship between the set of equilibria of a menu-game where lotteries are allowed, and that of the same game when stochastic menus are considered. We clarify this point in the following example.

There are two principals (denoted 1 and 2). The two decision spaces are both given by the set $[0,1]$. We allow principals to offer menus, that is, subsets of $[0,1]$. There is one agent, who chooses his preferred outcome in each list, say $y_{1}$ from principal 1 and $y_{2}$ from principal 2. Then, he communicates the choice to every principal, he says $y_{1}$ to principal 1 and $y_{2}$ to principal 2. In a final step, the actions $y_{1}$ and $y_{2}$ are implemented. In this example there is no moral hazard problem, since $E=\emptyset$ and we also assume that the agent has no private information.

The preferences of principal 1 over actions are defined as given by:

$$
V_{1}\left(y_{1}, y_{2}\right)=y_{1}\left(1-4 y_{2}\right) \text {. }
$$

Principal 2's utility function is

$$
V_{2}\left(y_{1}, y_{2}\right)=10-\left(\frac{1}{8}-y_{2}\right)^{2}
$$

The agent's preferences are given by

$$
U\left(y_{1}, y_{2}\right)=-\left(y_{1}-y_{2}\right)^{2} .
$$

We claim that the following strategies form an equilibrium:

- Principal 1 offers the menu $\{1 / 8\}$.

- Principal 2 offers the menu $[0,1]$

- The agent chooses $y_{2}=1 / 8$ 
In this situation, $U=0, V_{2}=10$ and $V_{1}=1 / 16$. Observe that Principal 1 offers a so-called degenerate menu, i.e. his menu is made of one allocation only.

We now argue that this strategy profile constitute an equilibrium. First, consider principal 1: he has no reason to deviate to another degenerate menu $\left\{y_{1}\right\}$. If he does that, then the agent has an incentive to choose the item $y_{2}=y_{1}$ in the principal 2's menu. As a consequence, the utility of the principal 1 becomes:

$$
V_{1}\left(y_{1}, y_{1}\right)=y_{1}\left(1-4 y_{1}\right) \text {. }
$$

that is maximized for $y_{1}=1 / 8$. Since principal 1 has no incentive to deviate to a degenerate menu, he will also not have any incentive to deviate to a more complex menu.

Principal 1 has a profitable deviation if lotteries are allowed. Suppose he plays the following lottery: $2 / 17$ with probability $1 / 3$ and $3 / 17$ with probability $2 / 3$, he can improve his payoff. In this case, the agent has to choose his preferred value of $y_{2}$ in the menu offered by the second principal which maximizes his expected utility. Simple computations show that the maximum of

$$
-\frac{1}{3}\left(y_{2}-\frac{2}{17}\right)^{2}-\frac{2}{3}\left(y_{2}-\frac{3}{17}\right)^{2}
$$

is attained when $y_{2}=\frac{8}{51}$ and thus the agent will choose $y_{2}=\frac{8}{51}$ in principal 2's menu. Finally, the utility of principal 1 is equal to

$$
\frac{1}{3} \frac{2}{17}\left(1-4 \frac{8}{51}\right)+\frac{2}{3} \frac{3}{17}\left(1-4 \frac{8}{51}\right),
$$

which is strictly greater than $\frac{1}{16}$.

\section{Discussion}

One should observe that at equilibrium menus are convex sets, and all players have standard utility functions. That is, the role of lotteries cannot be explained by existence of some trivial non-convexities in the game.

Let us foccus on the relationship between the agent and the first principal. They are Nash players and they take principal 2's strategy as given. Even if there is no moral hazard issue in our game, the agent takes a non-contractible action: he chooses the decision $y_{2}$ in the menu offered by the second principal. Thus, principal 1 is in fact acting as if he were in a moral hazard framework. He chooses $y_{1}$ and the agent chooses $y_{2}$. 
In is well known in moral hazard framework settings, lotteries may increase the payoff of the principal. Prescott (1999) gives two conditions under which a principal may have an incentive to offers lotteries instead of deterministic allocations. First, if utilities functions are not concave ${ }^{4}$, then the principal is likely to use stochastic mechanisms: if the agent is risk-seeker, there is an potential gain by using lotteries. But Prescott (1999) also indicates that non-separability between $y_{1}$ and $y_{2}$ in the agent's preferences makes stochastic mechanism desirable, even if the agent is risk-averse.$^{5}$ Our example is related to other results such as Cole (1989), and thus it could be interpreted in a similar way.

We also notice that lotteries play a critical role in contract theory. In multi-agent games Strausz (2003) has recently shown that the Revelation Principle does not hold for deterministic mechanisms, and direct mechanisms may be suboptimal. Lotteries are relevant in general equilibrium settings: Prescott \& Townsend (1984) have shown that lotteries may help to convexify principals' program and are necessary to show the existence of an equilibrium when asymmetric information is explicitly introduced. Not surprisingly, lotteries also play an important role in common agency games.

Lotteries are sometimes considered as unrealistic. Prescott (1999) provides some arguments against that view. If lotteries are not explicitly found in the terms of contracts, they can be indirectly implemented. Examples of such complex indirect stochastic mechanisms are given by Cole \& Prescott (1997) and Lehnert (1998).

\section{Conclusion}

The Delegation Principle is often invoked in applied papers to justify attention to menus. For sake of simplicity and because of their economic appeal, authors limit attention to pure strategy equilibria where competition is restricted to be in deterministic menus. ${ }^{6}$

However, the equilibrium outcomes of Common Agency games where competition is arbitrarily restricted to deterministic menus may be not robust to the introduction of stochastic mechanisms.

\footnotetext{
${ }^{4}$ With linear preferences, the solution is typically a corner solution and lotteries play no role.

${ }^{5}$ If agent's preferences are separable in $y_{1}$ and $y_{2}$, if the utility function $U\left(y_{1}, y_{2}\right)$ can be written as $\tilde{U}\left(\Phi\left(y_{1}\right), \phi\left(y_{2}\right)\right)$, then Attar et al. (2005) have shown that the Revelation Principal generically applies and that the restriction to direct mechanisms is without loss of generality.

${ }^{6}$ Examples are given by Biais et al. (2000), Martimort \& Stole (2003a), Martimort \& Stole (2003b), Calzolari (2004) or Bisin \& Guaitoli (2004).
} 


\section{References}

Attar, A., E. Campioni, G. Piaser, \& U. Rajan (2005) On multiple principal, multiple agent models of moral hazard. Université catholique de Louvain, Mimeo.

Attar, A., D. Majumdar, G. Piaser, \& N. Porteiro (2005) Common agency games with separable preferences. Université catholique de Louvain, Mimeo.

Biais, B., D. Martimort, \& J.-C. Rochet (2000) Competing mechanisms in a common value environment. Econometrica 78, 799-837.

Bisin, A. \& D. Guaitoli (2004) Moral hazard and non-exclusive contracts. RAND Journal of Economics 35, 306-328.

Calzolari, G. (2004) Incentive regulation of multinational entreprises. International Economic Review 45, 257-282.

Cole, H. L. (1989) Comment: General competitive analysis in an economy with private information. International Economic Review 30, 249-252.

Cole, H. L. \& E. C. Prescott (1997) Valuation equilibrium with clubs. Journal of Economic Theory 74, 19-39.

Lehnert, A. (1998) Asset pooling, credit rationing, and growth. Finance and Economics Discussion Series 1998-52. Washington: Board of Governors.

Martimort, D. \& L. A. Stole (2002) The revelation and delegation principles in common agency games. Econometrica 70, 1659-1673.

Martimort, D. \& L. A. Stole (2003) Contractual externalities and common agency equilibria. Advances in Theoretical Economics 3.

Martimort, D. \& L. A. Stole (2003) Market participation under delegated and intrinsic common agency games. Université des Sciences Sociales de Toulouse.

Peters, M. (2001) Common agency and the revelation principle. Econometrica 69, 1349-1372.

Prescott, E. C. \& R. Townsend (1984) Pareto optima and competitive equilibria with adverse selection and moral hazard. Econometrica 52, 21-45.

Prescott, E. S. (1999) A primer on moral hazard. Federal Reserve Bank of Richmond Economic Quarterly 85.

Strausz, R. (2003) Determininistic mechanisms and the revelation principle. Economics Letters 79, 333-337. 